\title{
Nonlinear hydrodynamic simulations of radial pulsations in massive stars
}

\author{
Catherine Lovekin ${ }^{1,2}$ and Joyce A. Guzik ${ }^{2}$ \\ ${ }^{1}$ Department of Physics, Mount Allison University \\ 67 York St., Sackville, NB E4L 1E6 Canada \\ email: clovekin@mta.ca \\ ${ }^{2}$ Los Alamos National Laboratory \\ XTD-NTA, MS T086, Los Alamos, NM 87545 USA \\ email: joy@lanl.gov
}

\begin{abstract}
We investigate the radial pulsation properties of massive main-sequence stars using both linear and non-linear calculations. Using 20,40,60 and 85 solar-mass models evolved by Meynet et al. (1994), we calculate nonlinear hydrodynamic envelope models including the effects of time-dependent convection. Many of these models are massive enough to lose a significant amount of mass as they evolve, which also reveals more helium-rich layers. This allows us to investigate the dependence of pulsation on mass, metallicity and surface helium abundance. We find that as a model loses mass, the periods become longer relative to the period predicted by the period-mean density relation (period $\times \sqrt{\bar{\rho}}$ is proportional to a constant, $Q$ ) for the initial model. Increased surface helium abundance causes a dramatic decrease in the period relative to that expected from $Q$, while changing the metallicity had little impact on the expected periods.
\end{abstract}

Keywords. stars: oscillations

\section{Introduction}

Many stars in the upper left quadrant of the H-R diagram are known to be pulsating (see, for example, Pamyatnykh 1999, Moffat 2010, Blomme et al. 2012, McNamara et al. 2012). In many cases, the evolution of these massive stars is not well understood, and pulsation characteristics can be used to constrain the evolutionary sequence. For this reason, we investigate radial pulsation in stars from 20 to $85 M_{\odot}$, and look at how the pulsation periods and amplitudes are affected by changes in mass, metallicity and surface helium abundance. We use parameters taken from a grid of stellar models published by Meynet et al. (1994) to calculate linear, non-adiabatic (LNA) pulsations in envelope models. The zoning in these models was arranged such that the driving region near $2 \times 10^{5} \mathrm{~K}$ was sufficiently resolved, and the zones extended to depths at or below $2 \times 10^{6} \mathrm{~K}$ in order to capture the damping region. The LNA calculations are then used to initialize the linear hydrodynamic pulsation code DYNSTAR. The hydrodynamic code produces a radial velocity curve, from which growth rates and dominant pulsation periods are extracted. Details of the codes are discussed in more detail in Guzik \& Lovekin (2012).

\section{Results}

We find that our instability regions are in agreement with the $\beta$ Cep instability strip as found by Pamyatnykh (1999), and our hydrodynamic model amplitudes become very large for models at or beyond the Humphreys-Davidson limit (Humphreys \& Davidson 1994). 


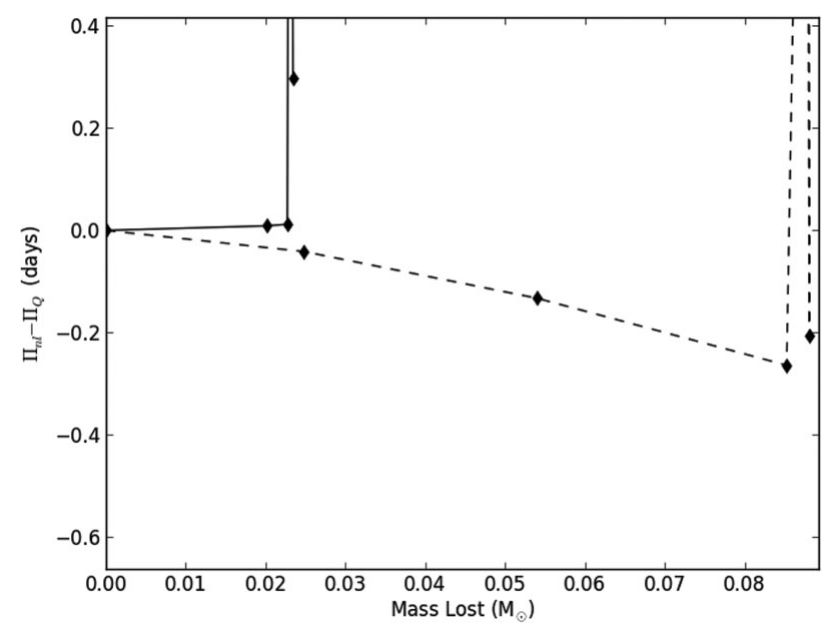

Figure 1. The difference between the nonlinear period and the $Q$ prediction as a function of mass lost in $40 M_{\odot}$ models with metallicity $Z=0.004$ (solid) and $Z=0.008$ (dashed). The sudden increase in the difference corresponds to the end of the main sequence in each model.

Our goal has been to investigate the changes in pulsation frequencies due to the effects of mass loss and changes in surface helium abundance as the models evolve. To do this, we use the $Q$ parameter $(=\Pi \sqrt{\bar{\rho}})$, which is approximately constant for models with similar structure. The periods of our models range from $<0.5 \mathrm{~d}$ for the main-sequence $20 M_{\odot}$ models, to approaching $80 \mathrm{~d}$ for the most massive evolved models. We find that, for our $20 M_{\odot}$ models, $Q$ can be used to predict the nonlinear pulsation frequencies quite well until the radius approximately doubles. We then applied the $Q$ parameter to the $40 M_{\odot}$ models, where the mass-loss rates are high enough to change the mass, but where the surface helium abundance stays nearly constant (Fig. 1). The spikes that appear around 0.02 and $0.09 M_{\odot}$ lost are the end of the main sequence, where the change in the structure of the model is large enough that $Q$ is no longer expected to be constant. In the most massive models, both mass loss and changes in surface helium abundance are significant. We find that an increase in the surface helium abundance decreases the periods relative to the predictions using $Q$. We also find that the magnitude of the decrease is much larger than the change produced by changes in metallicity or mass loss. We found that the metallicity had little effect on the periods.

We also investigated the amplitude of the pulsations in the hydrodynamic models, looking for trends with age, mass loss, surface helium abundance, and metallicity. We found that as the models lose mass, the amplitude of the pulsation increases, while increasing the surface helium abundance decreases the amplitude. The $20 M_{\odot}$ models that lose almost no mass show a slight trend of decreasing amplitude as the model ages. We did not find a clear trend with metallicity.

\section{References}

Blomme, R., Briquet, M., Degroote, P., et al. 2012, ASP-CS, 465, 13

Guzik, J. A. \& Lovekin, C. C. 2012, Astron. Review, 7, 13

Humphreys, R. M. \& Davidson, K. 1994, PASP, 106, 1025

McNamara, B. J., Jackiewicz, J., \& McKeever, J. 2012, AJ, 143, 101

Meynet, G., Maeder, A., Schaller, G., Schaerer, D., \& Charbonnel, C. 1994, A\&AS, 103, 97

Moffat, A. F. J. 2010, Highlights of Astronomy, 15, 366

Pamyatnykh, A. A. 1999, AcA, 49, 119 